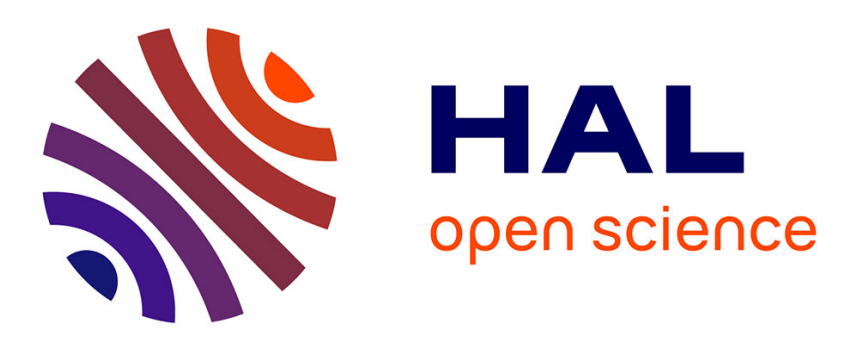

\title{
De l'Accueil des Réfugiés à la Gestion des Migrations. Les Îles du Dodécanèse: une Zone Tampon à Fort Potentiel entre la Grèce et la Turquie
}

Ioannis Georgikopoulos

\section{- To cite this version:}

Ioannis Georgikopoulos. De l'Accueil des Réfugiés à la Gestion des Migrations. Les Îles du Dodécanèse : une Zone Tampon à Fort Potentiel entre la Grèce et la Turquie. Anatoli, 2018, 9, pp.95-107. hal02466373

\section{HAL Id: hal-02466373 \\ https://hal.science/hal-02466373}

Submitted on 4 Feb 2020

HAL is a multi-disciplinary open access archive for the deposit and dissemination of scientific research documents, whether they are published or not. The documents may come from teaching and research institutions in France or abroad, or from public or private research centers.
L'archive ouverte pluridisciplinaire HAL, est destinée au dépôt et à la diffusion de documents scientifiques de niveau recherche, publiés ou non, émanant des établissements d'enseignement et de recherche français ou étrangers, des laboratoires publics ou privés. 


\section{De l'accueil des réfugiés à la gestion des migrations. Les îles du Dodécanèse : une zone tampon à fort potentiel entre la Grèce et la Turquie}

Ioannis Géorgikopoulos

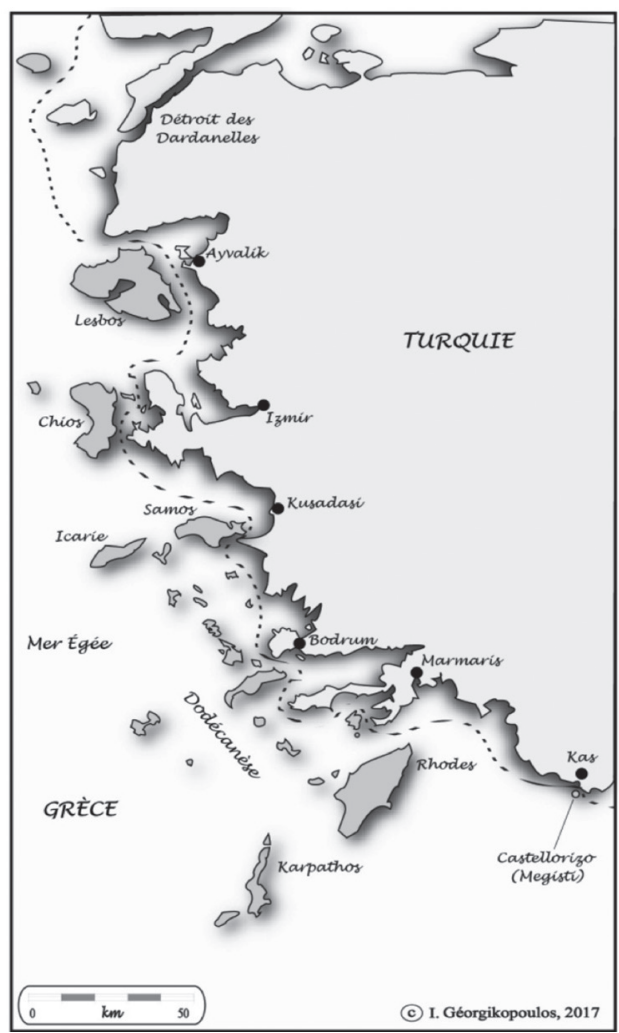

La frontière maritime gréco-turque

Foyers d'émigration jusqu'au début des années 1970, les îles du Dodécanèse se transforment en pôles d'immigration avec l'arrivée des migrants économiques égyptiens, pakistanais et afghans. À partir du début des années 1990, l'archipel accueille - comme le reste $\mathrm{du}$ territoire grec - de nombreux travailleurs albanais profitant de l'effondrement du régime de parti unique et de l'ouverture de la frontière (Sintès, 2010). Si les années 2000 ont vu la reprise des flux migratoires moyen-orientaux (Bathaïe, 2009), les arrivées massives et soudaines des réfugiés et migrants de provenances diverses ${ }^{1}$, traversant la frontière maritime gréco-turque en 2015, ont été, par son ampleur, un phénomène singulier pour la société dodécanésienne.

La proximité géographique des zones de tension (Proche et MoyenOrient), ainsi que les divergences

1. La Syrie, l'Irak et l'Afghanistan en étaient les principaux pays d'origine. 


\section{La Turquie et l’Europe. Études de terrain}

en ce qui concerne l'harmonisation des politiques européennes (d'entrée et d'asile) et la stratégie de dé-sécurisation mise en œuvre par la coalition gouvernementale grecque (Skleparis, 2017; Foucher, 2016) ont amené les îles à devenir, du jour au lendemain, les lieux de premier accueil pour les milliers de nouveaux arrivants. Les statistiques officielles produites nous donnent une image de la situation : entre le $1^{\text {er }}$ janvier et le 31 décembre 2015, 856723 personnes ont rejoint le territoire grec par la mer $^{2}$. Les îles proches de la côte turque, comme Lesbos, Chios, Samos, Farmakonissi, Agathonissi, Leros, Cos, Symi, Tilos, Rhodes et Castellorizo ont été les plus touchées par ce qui sera dénommé « crise migratoire».

L'augmentation quotidienne du nombre d'arrivants et l'absence d'un plan d'action solide, relatif à la capacité d'accueil et d'hébergement, ont momentanément déstabilisé les sociétés locales. Dans le Dodécanèse, la coopération entre l'armée grecque, l'Agence des Nations unies pour les réfugiés (UNHCR) et les organisations non gouvernementales (ONG) a apporté des solutions provisoires, essentiellement en ce qui concerne les besoins de première nécessité (hébergement, nourriture, protection des mineurs non accompagnés). Mais elle n'a pour autant affaibli ni les tensions internes aux sociétés insulaires touchées par la crise financière ni la particularité politicojuridique du franchissement de la frontière égéenne et toutes les complexités géopolitiques qui l'accompagnent.

Le premier choc fut pourtant vite dépassé. Face à de nouveaux défis venant confronter les îles à un environnement régional en mutation, les Dodécanésiens ont été appelés à réactiver leurs réflexes d'ouverture et à mobiliser les atouts hérités d'un passé riche et mouvementé. De par sa position géographique, sa diversité culturelle et son histoire ${ }^{3}$, 1'archipel semble être en mesure d'ajuster ses équilibres internes en fonction des réalités qui touchent sa démographie et son rôle géopolitique au sein d'une région de plus en plus instable. Ainsi, les compromis ayant lieu sans cesse au sein de ce carrefour géopolitique et les spécificités démographiques qui le caractérisent sont susceptibles d'apporter des réponses locales aux enjeux nationaux et européens qui se dessinent derrière le récent afflux d'immigration.

2. Voir le rapport annuel de l'Agence grecque pour les réfugiés (helliniko symvoulio gia tous prosfyges) du 20 avril 2016, ainsi que le rapport "Greece Refugee Emergency Response-Update \#8, 29 November-31 December 2015” de l'Agence des Nations unies pour les réfugiés.

3. Le Dodécanèse a été marqué par plusieurs annexions et occupations. Il porte, de ce fait, sur son paysage les traces de multiples présences : byzantine, latine, ottomane, italienne, britannique et grecque. 


\section{L'explosion des passages : l'archipel face à la pression migratoire}

En 2015, les flux migratoires ont pris un essor phénoménal. Les mutations géopolitiques au Moyen et au Proche-Orient (conflit turco-kurde, insurrections afghanes, crise syrienne) et l'émergence des groupes terroristes comme Daech sont venues s'additionner aux facteurs de mobilité déjà présents, tels que les écarts entre les niveaux de développement humain, les crises politiques, les catastrophes naturelles, le changement climatique etc. Le nouveau contexte régional et les arrivées massives ont déclenché une réaction phobique en chaîne, se manifestant à travers le durcissement des régimes frontaliers à l'intérieur de l'Union européenne (UE). La décision prise par l'Autriche de restaurer ses frontières avec l'Italie, la Slovénie et la Hongrie (février 2016) a été suivie par la fermeture en série de frontières nationales entre l'Allemagne et le Danemark, le Danemark et la Suède, la Belgique et la France, ainsi que par la fermeture de la « route des Balkans », empruntée par les réfugiés et les migrants pour rallier l'Est, l'Ouest et le Nord de l'Europe (Foucher, 2016). Ces évolutions ont aggravé la situation déjà difficile dans les pays de premier accueil comme la Grèce, où un grand nombre de réfugiés et de migrants continuent à être bloqués. Selon les rapports d'Amnesty International, les conditions de détention sont particulièrement préoccupantes et le délai de traitement des demandes d'asile auprès des autorités grecques continue à exiger une amélioration immédiate ${ }^{4}$. La cristallisation de cette situation a eu des répercussions importantes autant sur le devenir des populations en mobilité que sur le vécu des populations locales et les équilibres internes.

Dans le Dodécanèse, l'ambiance générale d'insécurité diffuse et la transformation soudaine de l'expérience publique locale (centres d'enregistrements, camps d'accueil et d'hébergement) - similaire à d'autres pays de premier accueil comme l'Italie (Blanchard et Rodier, 2016) - eurent des effets multiples. En premier lieu, un sentiment de confusion puis de frustration s'est installé chez les habitants. Il en résulta pour une grande partie de la population dodécanésienne un repli sur soi au détriment des traditions régionales d'insularité et de maritimité poussant, elles, à l'ouverture. Les entretiens, menés à Cos et à Leros entre août et octobre 2015, dévoilent ce sentiment

4. Voir le rapport "Grèce 2016/2017», disponible sur: https:/www.amnesty.org/fr/countries/ europe-and-central-asia/greece/report-greece/. 


\section{La Turquie et l’Europe. Études de terrain}

ambigu dominant les visions spatio-temporelles des communautés locales et trouvant ses racines dans les flux et les reflux de la vie insulaire entre le retrait vers l'intérieur en cas de danger et la projection vers l'extérieur pour des raisons économiques (commerce, échanges, etc.) Ainsi, il n'est pas étonnant de rencontrer des habitants de l'île de Cos «conscients de leur devoir humanitaire », mais qui restent pour autant méfiants vis-à-vis des NonSyriens souvent perçus comme « des envahisseurs musulmans extrémistes et, potentiellement dangereux». Les propos de Mme S., sexagénaire habitante de l'île de Leros, restent parmi les témoignages les plus significatifs :

"Je ne comprends pas comment l'État a pu prendre la décision de laisser passer tout le monde. Nous, on a essayé d'aider en leur ouvrant nos portes et en donnant ce qu'on pouvait; mais je n'aime pas qu'ils rentrent "par la fenêtre". Si c'est mon choix, tant mieux; ceux qui pressent les autres à tout supporter par solidarité tandis qu'eux, ils ne font rien, qu'ils se taisent. Moi, j'ai voulu aider et je l'ai fait, des fois plus que je ne le pouvais, mais au bout d'un moment je me sentais de plus en plus menacée. Aider qui en premier et jusqu'à quel point? Qui va nous aider le moment venu? La mer fait venir des enfants innocents, mais aussi des gens prêts à tout. Leros c'est une petite île qu'il faut protéger, pas une passerelle libre réservée à toute la misère du monde. »

Ensuite, la crise migratoire a donné une autre dimension au clivage politique interne, en mettant aux prises les différents acteurs impliqués sur place. Les sociétés locales se sont trouvées divisées entre tendances «sans frontières » et habitants plus réservés toujours sous le choc des arrivées massives. À Leros, les tensions entre un groupe "pro-migrant»- organisé par des militants envoyés depuis Athènes et rassemblant touristes et habitants bénévoles - et une municipalité méfiante proche des milieux économiques de l'île restent à l'ordre du jour. Outre les problèmes propres à leur situation, les immigrés doivent aussi subir une situation politisée à l'extrême, résultat de l'interaction entre clivages politiques nationaux et enjeux locaux.

Dans le même temps, à l'échelle de l'archipel, certains responsables politiques évoquent la nécessité d'obtenir des «microcrédits » de l'État et tentent de capter des financements européens et internationaux dans leurs circonscriptions législatives. Pour ce faire, ils avancent leur rôle primordial dans le succès de toute initiative portant sur la gestion de la situation afin de justifier leur implication bien que souvent superficielle. Ceci n'est pas sans rappeler les modalités du processus de la construction stato-nationale dans le Dodécanèse après son rattachement au territoire grec (1947), et surtout après 1980 (Géorgikopoulos, 2016). À partir de 1947, l'introduction du système clientéliste et l'activation nécessaire - étant donné le climat instable de l'époque (Guerre civile grecque) - des mécanismes de centralisation furent 


\section{De l'accueil des réfugiés à la gestion des migrations}

associées à une forte dépendance politico-économique de l'archipel vis-à-vis d'Athènes. Les années 1980 et le nouveau contexte européen (adhésion de la Grèce en 1981) ont, par la suite, donné l'occasion aux élites locales de développer plusieurs stratégies d'accaparement des ressources financières par le biais des subventions ou encore pour promouvoir, sur place, la ligne partisane, ce qui joua un rôle important dans le dépassement du clivage centre/ périphérie. La nouvelle donne migratoire et sa forte médiatisation ont rappelé à certaines élites locales l'importance géopolitique de leurs circonscriptions sur fond d'espoir de l'apport d'une nouvelle rente.

Mais la transformation, à partir de 2010, des politiques européennes à l'égard de l'octroi des financements et les contrôles introduits dans la gestion des programmes ont réduit sensiblement la marge de manœuvre politique à l'échelle locale. En effet, un des problèmes majeurs à l'heure actuelle n'est pas l'absence de fonds destinés à l'amélioration de la situation ${ }^{5}$ mais leur absorption par le ministère grec de la politique migratoire et par les municipalités impliquées. Selon les données de la Commission européenne ${ }^{6}$, des 908,6 millions d'euros (programme long terme 2014-2020 de 537,6 millions et fonds d'urgence de 371 millions) engagés pour l'aide humanitaire et le financement des politiques nationales de gestion de la situation, seulement 398 millions ont pu être décaissés entre 2015 et 2017. La cause principale en est le non-respect - du côté des instances nationales et locales grecques - des obligations et des délais définis par les institutions européennes, et plus particulièrement le manque de moyens administratifs et de capacité structurelle nécessaires, les processus opaques de recrutement de personnel, ainsi que le retard dans le dépôt des dossiers complets auprès de la Commission européenne ${ }^{7}$.

Dans ce contexte, une observation plus attentive de situations quotidiennes nous permet d'évoquer un lien entre les effets internes de la crise

5. À titre indicatif, rappelons l'existence du «Fonds Asile, Migration et Intégration» (AMIF) et le «Fonds pour la Sécurité Intérieure» (ISF) de l’UE pour la période 2014-2020, comprenant, entre autres, le fonds pour les réfugiés (FER), le fonds pour les frontières extérieures (FFI), le fonds d'intégration (FEI), et le fonds pour le retour (FR).

6. Voir: "Managing Migration. EU Financial Support to Greece", 17 octobre 2017, disponible sur: https://ec.europa.eu/home-affairs/what-we-do/policies/european-agenda-migration/ background-information_en.

7. Voir : «Les réfugiés en Grèce : défis et risques - Une responsabilité européenne », Résolution 2118 (2016) de l'Assemblée parlementaire du Conseil de l’Europe; Howden, D., Fotiadis, A., «The Refugee Archipelago: The Inside Story of What Went Wrong in Greece», Refugees Deeply, 6 mars 2017; le quotidien grec Kathimerini du 15 octobre 2017. 


\section{La Turquie et l'Europe. Études de terrain}

financière et la maîtrise des bouleversements externes. Selon mes recherches menées in situ, un grand nombre d'entrepreneurs ont profité de l'arrivée massive des migrants et surtout des réfugiés syriens - le plus souvent possédant des actifs (bijoux, portables, argent liquide) d'une valeur considérable - ainsi que de la forte présence des ONG et des reporters. Lors de notre rencontre à Leros, M. M., restaurateur à Lakki ayant juste dépassé la quarantaine, $\mathrm{m}^{\prime}$ informe :

"Les entrepreneurs leriotes disent souvent qu'ils ne supportent plus les migrants et que cela affecte l'image touristique de l'île; pourtant, entre septembre 2015 et janvier 2016, la plupart d'entre eux n'ont fait que profiter de la situation. Afin de signer les conventions avec l'État sur la préparation de nourriture pour les migrants et empocher les cinq euros prévus par personne nourrie, beaucoup de restaurateurs ont distribué du riz avec du ketchup! C'était la honte. De plus, quand ils ont vu les Syriens sortir les billets de cinq cents euros, les entrepreneurs et les commerçants ont trouvé l'occasion recherchée : aux tabacs, ils effaçaient les prix des cigarettes pour les vendre à sept ou huit euros le paquet; ils ont même mis un prix pour le chargement des téléphones portables des migrants dans les cafés. Un grand nombre d'hôtels, prêts à fermer à cause de l'endettement et de la taxation, ont aussi trouvé des clients parmi les réfugiés mais aussi parmi les membres des différentes ONG venus en aide. Les tarifs des nuitées variaient selon la situation économique de l'hôtelier.»

La création d'un centre d'enregistrement (hotspot) à Lépida, au sein de l'ancien hôpital psychiatrique de l'île, ainsi que l'hébergement des mineurs non accompagnés dans l'ancien bâtiment du Pikpa (fondation d'accueil social et de soins médicaux), suite aux initiatives de la société civile locale, ont offert un cadre protecteur aux migrants et réfugiés sur place.

Depuis mars 2016, l'accord UE-Turquie - prévoyant la gestion de l'immigration irrégulière et la réadmission conditionnée en Turquie de migrants et réfugiés orientaux arrivés en Grèce - semble, malgré les controverses, tarir les flux ${ }^{8}$. Néanmoins, les îles grecques continuent à constituer une zone tampon entre la Turquie et la Grèce, où reste bloqué un nombre considérable de nouveaux arrivants. La présence de 10904 personnes dans les camps de Lesbos, Chios et Samos et de 4354 personnes dans les camps des îles du Dodécanèse ${ }^{9}$ constitue un vrai défi auquel les sociétés locales sont appelées à répondre. Malgré les difficultés, le dépassement du premier choc et l'évolution des interactions entre les habitants et une partie des populations en mobilité

8. Passage de 856723 arrivées par la mer Égée en 2015 à 24739 en 2017 , selon les données de l'UNHCR au 10 novembre 2017.

9. Selon le rapport de l'UNHCR, le $1^{\text {er }}$ août 2017. 
semblent mettre en perspective le fort potentiel du monde insulaire grec en matière d'intégration de nouveaux éléments, et réactiver les anciens réflexes d'ouverture. À cet égard, le Dodécanèse présente des caractéristiques démographiques et culturelles qui, exprimant une combinaison d'éléments de continuité et de rupture, sont susceptibles de répondre aux exigences aussi bien internes qu'externes du fait migratoire.

\section{Les constantes de l'histoire démographique du Dodécanèse comme atouts pour l'intégration}

De par sa position de carrefour, l'archipel a toujours fait l'objet d'une démographie fluctuante. Inscrit dans une logique de réseaux, le Dodécanèse a participé aux grandes vagues migratoires qui ont marqué la région à travers le temps. Pendant la période ottomane (1522-1912), les transferts des populations issues des territoires impériaux (pratique de sürgün) avaient diversifié le paysage socioculturel du Dodécanèse - majoritairement chrétien (orthodoxes et catholiques) - en y introduisant des communautés musulmanes, arméniennes et juives (Kasperson, 1966). La cohabitation et l'interaction, en général paisible, entre les différents groupes religieux ont conduit au développement des sociabilités commerçantes, ainsi qu'à une prospérité économique et à une richesse culturelle notables. Plus particulièrement, l'essor de la communauté orthodoxe (Rum millieti) et sa projection vers l'extérieur par le biais des activités commerciales lui ont permis de tisser des liens socio-économiques avec la région élargie de l'archipel (Égypte, Anatolie), mais aussi avec l'Europe occidentale de l'époque. Ces liens faciliteront par la suite l'installation des émigrés insulaires de la fin $\mathrm{du} \mathrm{XIX}^{\mathrm{e}}$ siècle et, par voie de conséquence, la création d'une première diaspora dodécanésienne orthodoxe.

La modernité introduite par l'occupation italienne (1912-1943) n'apporta pas - en tout cas jusqu'en 1937, année de durcissement du régime fasciste dans les îles (remplacement du gouverneur Mario Lago par Cesare Maria de Vecchi et prise en main des instances culturelles, adoption graduelle des lois raciales) - de changements majeurs dans l'organisation sociale et l'autogestion communautaire, surtout dans les espaces ruraux de l'archipel. Malgré ce fait, et bien que les communautés juives et musulmanes aient maintenu presque intact le nombre de leurs membres, la communauté orthodoxe vit ses effectifs diminuer de temps à autre. Le changement de conjoncture et le passage difficile 


\section{La Turquie et l’Europe. Études de terrain}

à un cadre centralisé activèrent des flux d'émigration successive des Grecs dodécanésiens vers l'Égypte, les États-Unis, le Canada, l'Amérique du Sud et l'Australie (Kasperson, 1966), mobilités rejoignant les grandes vagues migratoires issues du territoire grec entre 1900 et 1921 (Bruneau, 1992). Toutefois, le vide fut comblé par l'arrivée des colons italiens et levantins, venant peupler les centres urbains tels que les villes de Rhodes et de Cos. L'adoption des lois raciales en 1938 puis l'occupation allemande des îles (1943-1945) ont conduit à la disparition presque totale des juifs dodécanésiens qui n'ont pas choisi la voie de l'émigration: entre 1940 et 1947 la communauté passe de 2038 à 49 personnes (Doxiadis, 1947) et en 1951 elle compte seulement 34 membres $^{10}$. Les Dodécanésiens musulmans, quant à eux, ont pu maintenir leur présence dans les îles de Rhodes et de Cos. Territoire italien pendant la guerre gréco-turque de 1919-1922, le Dodécanèse n'avait pas participé aux échanges de populations prévus par le Traité de Lausanne (1923) entre Turquie et Grèce. De plus, contrairement aux juifs de l'archipel, les Dodécanésiens musulmans n’ont pas subi des déportations ou persécutions pendant la Seconde Guerre mondiale. C'est ainsi qu'au sortir de la Guerre, la communauté comptait 6230 personnes et passa à 4937 en $1951^{11}$.

Aujourd'hui, la cohabitation entre habitants orthodoxes et environ 5000 musulmans (3000 à Rhodes et 2000 à Cos) d'origine ottomane - devenus citoyens grecs au moment du rattachement des îles à la Grèce fait preuve de la forte capacité de compromis de la société dodécanésienne et contribue de manière significative à la cohésion socioculturelle interne. Cette spécificité démographique, doublée d'une scénographie multiculturelle (mosquées, églises, synagogues, cimetières), peut constituer un avantage comparatif pour l'accueil de nouveaux éléments, la situation résiduelle (préservation de la diversité ottomane) de l'archipel créant un contexte favorable pour l'intégration des immigrés musulmans, sans que ceux-ci soient brutalement éloignés de leurs attaches culturelles initiales. Ainsi, le Dodécanèse pourrait devenir un premier foyer d'adaptation des immigrés dans la société grecque, ce qui faciliterait aussi le passage graduel au contexte européen.

De surcroît, la spécificité démographique de l'archipel ainsi que le souvenir des moments difficiles ayant marqué sa démographie (exodes consécutifs de la jeunesse pour échapper à la précarité et l'instabilité de la vie insulaire jusqu'aux années 1970), confèrent aux habitants une grande flexibilité et une forte capacité d'adaptation aux nouveaux défis. Le nouveau

10. Selon le recensement de 1951. Source: Agence grecque de la statistique (ELSTAT).

11. Ibid. 


\section{De l'accueil des réfugiés à la gestion des migrations}

courant d'émigration dodécanésienne ${ }^{12}$ et le recul démographique qui en résulte, de concert avec le vieillissement de la population ${ }^{13}$, rendent encore plus urgente la réactivation des réflexes d'ouverture et d'intégration de nouveaux éléments. Dans ce contexte, et après une période tourmentée, les sociétés locales semblent reprendre conscience de leurs qualités et faire de l'expérience migratoire une véritable opportunité. À cet égard, la petite île de Tilos, comptant à peine 780 résidents permanents ${ }^{14}$, constitue un exemple éloquent ${ }^{15}$. Motivée et facilitée par la société locale et l'ONG grecque «Solidarity Now», l'installation des réfugiés syriens commence à contribuer de façon très positive à la vie quotidienne, les chefs de la municipalité et les habitants ayant très vite compris le rôle que ces arrivées pouvaient jouer dans le renouveau démographique et le développement économique de l'île. Cette évolution n'a pas échappé à la presse européenne ; le 22 octobre 2017, le quotidien «La Libre Belgique» publie un reportage sous le titre: «Tilos, l'île sauvée par les réfugiés ${ }^{16} »$.

La société dodécanésienne semble ainsi revisiter les constantes de l'histoire et de l'actualité démographique de l'archipel pour redynamiser l'économie et la cohésion sociale à l'échelle locale. En tant que frontière entre la faible démographie européenne ${ }^{17}$ et la zone dynamique de $l^{\prime}$ Afrique du Nord et de l'Asie de l'Ouest (Zaninetti, 2017), le Dodécanèse présente un fort potentiel à moyen et à long terme dans la mesure où il peut faire l'articulation entre l'Europe occidentale et les réalités qui se dessinent sur ses pourtours Sud-Est. En tant que formes de prémodernité introduites dans la postmodernité, l'organisation réticulaire des mobilités (flux d'émigration et d'immigration), la diversité culturelle et la forte capacité de compromis internes deviennent la contribution de la tradition méditerranéenne - dans laquelle s'inscrit l'archipel - à la réorganisation des conceptions et à la mise en œuvre des politiques adaptées.

12. Avec plus de 6000 personnes, sur une population de 29452 habitants (recensement de 2011), ayant émigré vers l'Australie (Darwin), les États-Unis (Tarpon Springs) et le Sud de France (Portde-Bouc et Port-Saint-Louis-du-Rhône) entre 2011 et 2015, l'île de Kalymnos constitue l'exemple le plus marquant.

13. Entre 2001 et 2011, la population jeune (o-39 ans) de l'archipel a baissé de 8,3\%. Source : ELSTAT.

14. Recensement de 2011. Source : ELSTAT.

15. "Tilos, une petite île grecque de précurseurs", quotidien suisse Le Temps, 11 août 2017.

16. Disponible sur: http://www.lalibre.be/actu/international/tilos-l-ile-sauvee-par-les-refugies59ea2a31cd7095e2f7091907.

17. “World Population Prospects: The 2015 Revision, Key Findings and Advance Tables", Nations unies, Département des Affaires économiques et sociales/Division de la population. 


\section{Derrière le défi migratoire, des enjeux géopolitiques majeurs}

La crise migratoire de 2015 et la reprise des flux à partir de septembre 2017, démontrent la complexité des enjeux qui se dessinent à plus grande échelle. Au premier abord, le différend gréco-turc en mer Égée ne cesse, depuis 1974, de compliquer les relations diplomatiques entre les deux pays. Le contentieux apparaît comme l'avatar de l'affaire chypriote, l'invasion/intervention turque dans le nord de l'île ayant été suivie par une série de contestations à l'égard de la souveraineté grecque en mer Égée. L'extension de l'espace aérien (FIR) et la démilitarisation de certaines îles restent des problèmes épineux liés à la défense de l'espace maritime et insulaire grec. La particularité géographique de la mer Égée pose aussi problème quant à la largeur de la mer territoriale - dont l'extension fait toujours l'objet d'un casus belli turc - et à la Zone économique exclusive (ZEE) grecques, thèmes étroitement liés à la délimitation du plateau continental (Bertrand, 2003 ; Prévélakis, 2006). L'adhésion de la Grèce à la Communauté européenne (CEE) en 1981 a confirmé le statut des îles en tant que frontière externe de l'UE mais n'a guère changé la nature du contentieux bilatéral. En 1996, la crise des îlots d'Imia/Kardak dans le Dodécanèse a constitué l'incident le plus grave entre les deux pays et a failli se solder par une confrontation armée. Le minimum de stabilité maintenu dans les rapports gréco-turcs à partir des années 2000 n'empêche pas l'apparition régulière des incidents navals et aériens.

Les hauts et les bas dans les relations bilatérales ont créé un nouveau vide géopolitique qui engendre la multiplication des interventions extérieures dans les zones dites «grises». La situation difficile a interféré avec la marge de manœuvre des opérations, relatives au contrôle des côtes et du trafic en mer Égée (intercepter les passeurs, réguler les flux et bloquer les routes de l'immigration irrégulière), menées par l'Agence européenne de gardes-frontières (FRONTEX) et par l'Alliance atlantique (OTAN). En février 2016, les tensions entretenues sur la frontière aérienne et maritime ont conduit à un veto turc entraînant l'exclusion du Dodécanèse des activités dans la zone. Ce positionnement turc fait écho au rapprochement stratégique et énergétique entre Chypre, Israël, la Grèce et l'Égypte, fortement disputé par Ankara. Plus particulièrement, la «Déclaration du Caire » portant sur la délimitation des zones d'exploitation des champs gaziers et signée en 2014 a été directement contestée par la Turquie. La tension a été ravivée lorsque, en janvier 2016, la Grèce, Chypre et Israël ont annoncé leur coopération sur le projet de gazoduc « EastMed », destiné à exporter à terme du gaz naturel de Méditerranée orientale vers l’Europe. 


\section{De l'accueil des réfugiés à la gestion des migrations}

En mars 2016, le pacte migratoire entre l'UE et le gouvernement turc, à l'initiative de la chancelière allemande, a fait l'objet de plusieurs critiques ${ }^{18}$. Parmi les dispositions figurent une assistance financière de 6 milliards d'euros pour que la Turquie reprenne les demandeurs d'asile arrivés en Grèce après la signature de l'accord, ainsi que des engagements de la part de l'Union en vue d'une reprise des négociations au sujet de l'adhésion de la Turquie et de la possibilité de la suppression du régime des visas pour ses ressortissants voyageant en Europe. À la mi-juillet 2016, le coup d'État manqué et le virage de la diplomatie turque ont changé la donne et ont soulevé de graves problèmes, qui laissent planer une grande incertitude sur le devenir des rapports turcoeuropéens (Kundnani et Ziebarth, 2017). Suite aux malentendus réciproques à propos des conditions assurant la pérennité de l'accord UE-Turquie, liés notamment à l'adaptation de la législation antiterroriste du pays aux standards européens, les flux ont repris depuis septembre 2017. Bien qu'acteur important dans la gestion de la situation, la Turquie n'a pas le monopole sur les tentatives d'instrumentalisation du défi migratoire. Dans la tourmente des négociations financières suivant l'arrivée au pouvoir de la coalition gouvernementale (alliance entre gauche radicale et droite souverainiste) en Grèce, le nouveau ministre de la défense avait menacé, en mars 2015, d' «inonder l'Europe de migrants, dont des personnes affiliées à Daech» dans le cas de non-accord avec les créanciers ${ }^{19}$, propos répété le mois suivant. Le lancement et le renforcement des patrouilles par des agences supranationales (FRONTEX, OTAN) et le partenariat UE-Turquie en fut la réponse tardive.

Le fait migratoire devient ainsi un outil de pression politique qui influe sur les rapports interétatiques (rivalités de pouvoir pour le contrôle des zones d'influence) et supra-étatiques (UE) en renforçant le rôle des zones tampons. Dans ce contexte, le Dodécanèse se trouve au centre des enjeux transcalaires qui se dessinent dans la région. Au même moment, il fait partie des projets russes et chinois de pénétration stratégique et économique en Méditerranée orientale, ce qui entraîne de nouvelles compétitions géopolitiques pour la défense des intérêts occidentaux dans cet espace frontalier. Ainsi, le regain de visibilité que connaît l'archipel ces dernières années est susceptible de constituer le premier pas vers la prise de conscience de l'importance des

18. Voir, entre autres : «Accord UE-Turquie : la honte de l'Europe», Amnesty International, 17 mars 2017. 19. «Athènes menace d'envoyer ses immigrés à Berlin », Le Figaro, 9 mars 2015 ; "Greece's Defence Minister Threatens to Send Migrants Including Jihadists to Western Europe », The Telegraph, 9 mars 2015 ; “Greece threatens to unleash wave of migrants on the rest of Europe 'including Isis jihadists", The Independent, 10 mars 2015. 


\section{La Turquie et l'Europe. Études de terrain}

périphéries maritimes dans l'articulation entre les différentes échelles géographiques, allant du global au local.

\section{Conclusion}

Le cas du Dodécanèse permet de mettre en lumière le rôle politique de la mer et le potentiel du facteur local comme sources d'opportunités plutôt que de contraintes. De par sa position de carrefour, l'archipel est capable de combiner efficacement plusieurs réalités qui permettent de comprendre d'une autre manière les enjeux actuels directement ou indirectement liés aux mouvements des populations. Le souvenir toujours vivant de la géohistoire de la territorialité ottomane, le contact avec la culture méditerranéenne d'ouverture à la mer, l'influence occidentale et le vécu quotidien lié à la gestion de la situation frontalière sont les atouts dodécanésiens susceptibles de permettre à la Grèce d'avancer dans le sens de la construction d'une nouvelle image qui mettra en valeur les talents traditionnels de ses composantes. Le pays pourrait ainsi abolir son image négative et contribuer pleinement au sort du continent, en aidant à trancher dans le sens géopolitique et à redécouvrir la part orientale de la géohistoire européenne. À cet égard, le Dodécanèse joue un rôle significatif puisqu'il devient le lieu par excellence où se joue l'articulation entre les défis de la géopolitique interne et ceux de la géopolitique externe. Reste à voir à quel point la mise en valeur des qualités de l'archipel arrivera à compenser, sur le long terme, l'incertitude du contexte régional; d'ici là, l'expérience du passé peut continuer à nourrir des espoirs considérables.

\section{Références bibliographiques}

Antonopoulos, G. A., Winterdyk, J., "The smuggling of migrants in Greece: An examination of its social organization", European Journal of Criminology, vol. 3, n ${ }^{\circ}$, 2006, p. 439-461.

BатнаїЕ, A., «La Grèce, une étape cruciale dans le parcours migratoire des Afghans depuis la frontière iranienne jusqu'en Europe ", Méditerranée, n 113, 2009, p. 71-77.

Bertrand, G., Le conflit helléno-turc, Paris, Maisonneuve et Larose, 2003.

Blanchard, E., Rodier, C., " "Crise migratoire" : ce que cachent les mots », Plein droit, vol. 111, no 4, 2016, p. 3-6. 


\section{De l'accueil des réfugiés à la gestion des migrations}

BRUneAU, M., «L'Hellénisme. Un paradoxe ethno-géographique de la longue durée », Géographie et Cultures, n² 2, 1992, p. 45-74.

Cattaruzza, A., " "Zones grises", interstices durables de la carte politique ? Relecture critique d'un concept géopolitique », Bulletin de l'Association des Géographes Français, vol. $89, \mathrm{n}^{\circ} 1,2012$, p. 104-120.

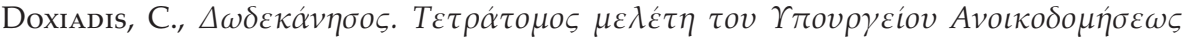
$\kappa \alpha \iota \sigma v v \varepsilon \rho \gamma \alpha \tau \omega v \tau$ Tov (Dodécanèse. Étude du ministère de la Reconstruction et de l'Urbanisme), vol. 1-4, Athènes, 1947.

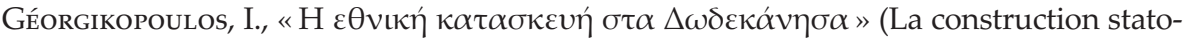

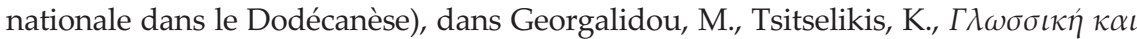

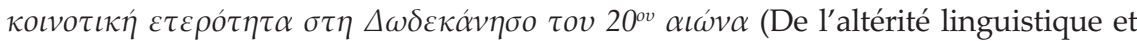
communautaire dans le Dodécanèse du xxe siècle), Athènes, Papazisis, 2016, p. 75-96.

Foucher, M., Le retour des frontières, Paris, CNRS éditions, 2016.

Kasperson, R. E., The Dodecanese: Diversity and unity in island politics, Research Paper $\mathrm{n}^{\mathrm{o}}$ 108, Chicago, The University of Chicago, 1966.

Kundnani, H., Ziebarth, A., «Entre l'Allemagne et la Turquie, l'enjeu des réfugiés », Le Monde diplomatique, $\mathrm{n}^{\circ}$ 754, janvier 2017, p. 16-17.

Pillant, L., « Les conséquences socio-spatiales des nouvelles modalités du contrôle migratoire à la frontière gréco-turque », L'Espace Politique [En ligne], vol. 25, no 1, 2015. URL : http://espacepolitique.revues.org/3369, Doi : 10.4000/espacepolitique.3369

PrévéLakis, G., Qui sont les Grecs ? Une identité en crise, Paris, CNRS éditions, 2017.

Prévélakis, G., Géopolitique de la Grèce, Paris, Bruxelles, Complexe, 2006 (2édition).

SchnApPer, D., «Les conséquences de l'immigration sur la société française », Cahiers français, $\mathrm{n}^{\circ} 383,2014$, p. 35-40.

Scol, J., "Développement touristique et dynamiques démographiques en Grèce: l'exemple du département insulaire du Dodécanèse », Espace, populations, sociétés, $\mathrm{n}^{\circ} 2$, 2003, p. 277-290.

Shachar, N., The Lost World of Rhodes: Greeks, Italians, Jews and Turks between Tradition and Modernity, Brighton \& Eastbourne, Sussex Academic Press, 2013.

Sintès, p., "Construction des discours d'appartenance en migration : l'exemple des Albanais en Grèce », Anatoli, n 1, 2010, p. 195-214.

SKLEPARIS, D., "'A Europe without walls, without fences, without borders': a desecuritisation of migration doomed to fail", Political Studies, 2017 [Publication anticipée en ligne], Doi: 10.1177/0032321717729631.

Wintol de Wenden, C., «Une nouvelle donne migratoire », Politique étrangère, n 3, 2015, p. 95-106.

Zaninetti, J.-M., Géographie des peuplements et des populations. L'homme sur la terre, Paris, PUF, 2017 (4édition). 
